gleichzeitig Vor- und Nachteil dieses Studiendesigns ist und Anlass zu weiteren Studien diesbezüglich geben sollte. Ob es sich hierbei um einen pharmakokinetischen oder pharmakodynamischen Effekt handelt, bleibt unklar. Insbesondere bei weit verbreiteter Gabe als Nahrungsergänzungsmittel kann sich im klinischen Alltag eine Überprüfung der Medikamente eines mit Lamotrigin behandelten Patienten lohnen.

Zusammengefasst liefert die Studie eine wichtige Ergänzung zu Behandlungsmöglichkeiten bei Patienten mit bipolarer Störung und depressiven Symptomen. Größer angelegte, industrieunabhängige Studien sind dringend notwendig, um diese ersten positiven Ergebnisse zu replizieren. Sowohl Quetiapin als auch Lamotrigin sind in unterschiedlichen Empfehlungsgraden zur phasenspezifischen Therapie der bipolaren
Depression in Deutschland bereits zugelassen, die Kombinationstherapie Quetiapin/Lamotrigin kann eine sinnvolle Strategie darstellen.

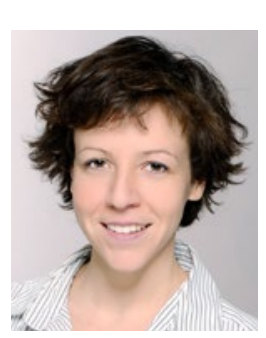

Dr. med. Alice Engel, Mainz

Klinik für Psychiatrie und Psychotherapie, Universitätsmedizin Mainz

E-Mail: alice.engel@unimedizin-mainz.de

\title{
Fibrillenstruktur beeinflusst das klinische Bild
}

Fragestellung: Mithilfe der ssNMR (Solid-state-Kernspinresonanzspektroskopie) untersucht die Studie die Struktur von Alzheimer-Fibrillen, die aus $\beta$-amyloidreichen Extrakten des zerebralen Kortex von an Morbus Alzheimer erkrankten Patienten gezüchtet werden.

Hintergrund: Amyloidfibrillen mit unterschiedlicher Tertiärstruktur unterscheiden sich in primären Neuronenkulturen erheblich in ihrer Toxizität. Exogen appliziertes $\beta$-Amyloid induziert bei transgenen Mäusen Amyloidablagerungen, die sich abhängig von der Herkunft des Materials unterscheiden. Größenverteilung und Widerstandsfähigkeit der Amyloidfibrillen gegenüber chemischer Denaturierung weisen zwischen Patienten mit schnell und langsam fortschreitendem Morbus Alzhei-

Qiang W, Yau WM, Lu JX et al. Structural variation in amyloid- $\beta$ fibrils from Alzheimer's disease clinical subtypes. Nature 2017; 541: $217-21$ mer deutliche Unterschiede auf. Einblicke in die Struktur der Amyloidfibrillen dürften grundlegende Implikationen für die Pathogenese, Diagnostik und Therapie der Alzheimer-Erkrankung haben.
Patienten und Methodik: Aus 37 kortikalen Gewebeproben von 18 Patienten mit typischem, langsam fortschreitendem Morbus Alzheimer ( $t-A D)$, posteriorer kortikaler Atrophie (PCA) und rapide fortschreitendem Morbus Alzheimer (r-AD) wurden $\beta$-Amyloidextrakte gewonnen und plattiert. Nach radioaktiver Markierung definierter Aminosäuren durch 15N und 13C wurden die sich entwickelnden Amyloidfibrillen mithilfe von TEM (Transmissionselektronenmikroskopie) und ssNMR analysiert.

Ergebnisse: Patienten mit t-AD und PCA bildeten einheitliche A $\beta 40$-Fibrillen aus, die durch eine periodische Modulation der Fibrillenbreite in Intervallen von $107 \pm 20 \mathrm{~nm}$ (Mittelwert \pm Standardabweichung) charakterisiert waren. Ein derart einheitliches Fibrillenmuster fand sich bei Patienten mit r-AD nicht. Die TEM wies ein Nebeneinanderexistieren verschiedenartiger Fibrillenstrukturen auf. Im Gegensatz zu A $\beta 40$ konnten für A $\beta 42$ keine einheitlichen Fibrillenmuster identifiziert werden.

Schlussfolgerungen: Die unterschiedliche Fibrillenstruktur dürfte Konsequenzen für die klinische Manifestation, die Diagnostik und Therapie des Morbus Alzheimer haben.

\section{- Kommentar von Dirk Hermann, Essen}

\section{Die Fibrillenstruktur könnte auch das Therapieansprechen beeinflussen}

Diese Studie ist die erste systematische Analyse des $\beta$-AmyloidFibrillenwachstums mit ssNMR, um unterschiedliche Phänotypen des Morbus Alzheimer zu charakterisieren. Basierend auf diesen Daten bleibt zu klären, in welcher Weise die beobachteten Fibrillenstrukturen die Entwicklung zerebraler $\beta$-Amyloidablagerungen beeinflussen. Es ist denkbar, dass verschiedene Amyloidfibrillen unterschiedlich auf Alzheimer-Therapeutika ansprechen. In jedem Fall dürften sich aus den Daten interessante Einblicke in Prozesse der Alzheimer-Propagation nach exogener $\beta$-Amyloidapplikation ergeben.

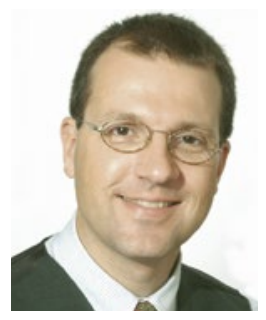

Prof. Dr. med. Dirk M. Hermann, Essen

Lehrstuhl für vaskuläre Neurologie, Demenz \& Altersforschung, Universitätsklinikum Essen E-Mail: dirk.hermann@uk-essen.de 